\title{
Pseudo-Differential Time-Domain Integrator Using Charge-Based Time-Domain Circuits
}

\author{
Arijit Karmakar ${ }^{\dagger}$, Valentijn De Smedt, Member, IEEE, and Paul Leroux, Senior Member, IEEE
}

\begin{abstract}
This work proposes a pseudo-differential timedomain integrator using half-delay time-domain registers and adders relying on charge-based time-domain circuits. It is implemented using a 65-nm CMOS Technology and performs first order integration of time-domain information within the range of [4 ns, $-4 \mathrm{~ns}$ ] across temperature $-40^{\circ} \mathrm{C}$ to $80^{\circ} \mathrm{C}$. It consumes 740 $\mu W$ with a supply voltage of $1.2 \mathrm{~V}$ at a $100 \mathrm{MHz}$ clock frequency. A delay-locked-loop (DLL) based foreground calibration is used to compensate for process and temperature variations.

Index Terms-Time-domain amplifier, time-domain adder, time integrator, half-delay, delay-locked-loop (DLL)
\end{abstract}

\section{INTRODUCTION}

The down-scaling of modern CMOS technologies has benefited the mixed-mode data converters utilising time-domain signal processing with improved time resolution. Although the converters have achieved fast operation with high throughput, but those using traditional voltage-domain signal processing suffer from an increased $1 / \mathrm{f}$ noise and reduced dynamic range due to scaled supply voltage. In time-domain signal processing, the circuits use the time-difference or interval between two discrete events to represent and process information. The basic time-domain blocks such as time-domain amplifiers help to improve the resolution by amplifying the time differences whereas the time-domain registers, adders and integrators assist in filtering [1] and data conversion applications [2]-[4]. In popular practice, the time-domain information is integrated in the phase-domain by using voltage-controlled-oscillators (VCOs), but suffers from VCO non-linearity and harmonic distortions. Although these can be reduced by using the VCOs inside $\Delta \Sigma$ loops, the overall performance is limited by the VCO phase noise. The time-domain integration could also be performed using bi-directional gated delay lines (BDGDL) [5] but gets affected from timing skew errors. Alternate approaches use charge-based time-domain amplifiers or adders [6], [7]. There the time-domain information is processed by exponential discharging of capacitors, introducing harmonic distortion as a result of the non-linear discharging profile. On the contrary, the methods using constant-current charging profiles [8] provide better linearity and less harmonic distortion.

\section{Proposed Time-Domain DifFEREnTial InTEGRAToR}

The proposed time-domain differential integrator (Fig. 1) operates by accumulating time-intervals over the sampling

The authors are with the ESAT-ADVISE research lab, KU Leuven, Belgium. †e-mail: arijit.karmakar@kuleuven.be.

This work is funded by the European's Union Horizon 2020 Research and Innovation Programme under grant agreement number 721624 'RADSAGA'. clock cycles similar to first order integration in discrete-time voltage-domain signal processing.

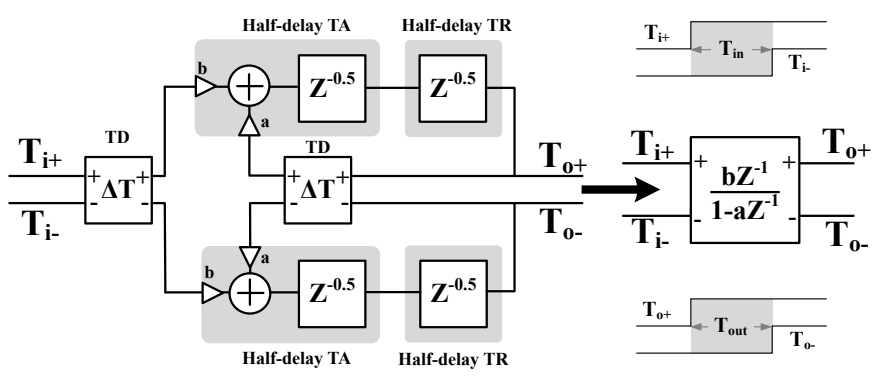

Fig. 1: Overview of the proposed time-domain integrator.

The time-domain integrator is implemented using timedifference detectors (TD), half-delay time-domain adders (TA) and time-domain registers (TR). The TD at the input is used to sample $T_{\text {in }}$ and that at the output is used to sample $T_{\text {out }}$ and provide feedback to the TA. Now, it can be derived for each clock cycle ' $\mathrm{n}$ ', $T_{\text {out }}[n]=b T_{\text {in }}[n-1]+a T_{\text {out }}[n-1]$, where $b$ and $a$ represents the signal-path gain and feedbackpath gain respectively. Then the system transfer function $H(z)$ can be expressed as, $H(z)=b z^{-1} /\left(1-a z^{-1}\right)$. Following the Forward Euler approximation, it can be written as, $H(s)=$ $b /\left(1-a+s T_{s}\right)$ where $T_{s}$ is sampling time period. For an input signal frequency $\Omega_{i n}$, the gain and phase of the integrator are $\frac{|b|}{\sqrt{(1-a)^{2}+\left(\Omega_{i n} T_{s}\right)^{2}}}$ and $-\tanh \frac{\Omega_{i n} T_{s}}{b(1-a)}$ respectively.

\section{CiRCUit IMPlEMENTATION}

\section{A. Half-delay Time-domain Register}

The half-delay time-domain register (TR) (Fig. 2(a)) is designed based on a constant-current charging-based timedomain amplifier [8], such that $T_{o}=T_{i} z^{-0.5}$ where $T_{i}$ and $T_{o}$ are the input and output pulse-time intervals respectively. As shown in Fig. 2(c), $T_{C L R}$ discharges the capacitor $C$ at each rising edge of the $C L K$. When $C L K=1, I_{1}$ charges $C$ for the time $T_{i}$ and thereafter $C$ holds the charge $\left(\Delta Q_{H}=I_{1} T_{i}\right)$ deposited. When $C L K=0, I_{1}$ again starts to charge $C$. The buffer at the output node of $C$ switches when the voltage across $C, V_{C}$ crosses $V_{T H}$, the switching threshold voltage of the buffer. The charge stored during $C L K=0$ till $V_{C}$ crosses $V_{T H}$, equals to $\Delta Q_{L}=I_{1}\left(T_{C L K} / 2-T_{o}\right)$. Now $I_{1}$ is set such that $\left(I_{1} / C\right)\left(T_{C L K} / 2-T_{C L R}\right)=V_{T H}$. Then for each clock cycle, $\frac{\Delta Q_{H}}{C} z^{-0.5}+\frac{\Delta Q_{L}}{C}=V_{T H}$ and the output $T_{o}$ can be derived as,

$$
T_{o}=T_{i} z^{-0.5}+T_{C L R}
$$




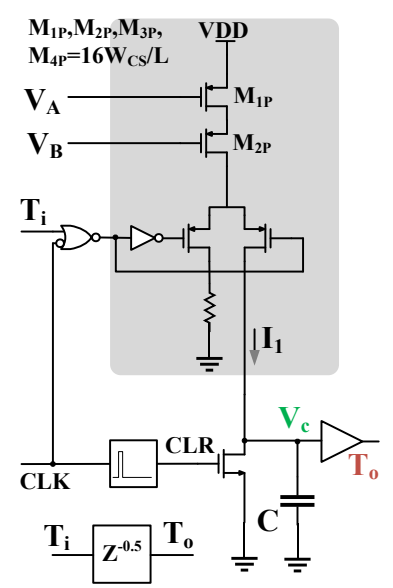

(a) Half-delay TR

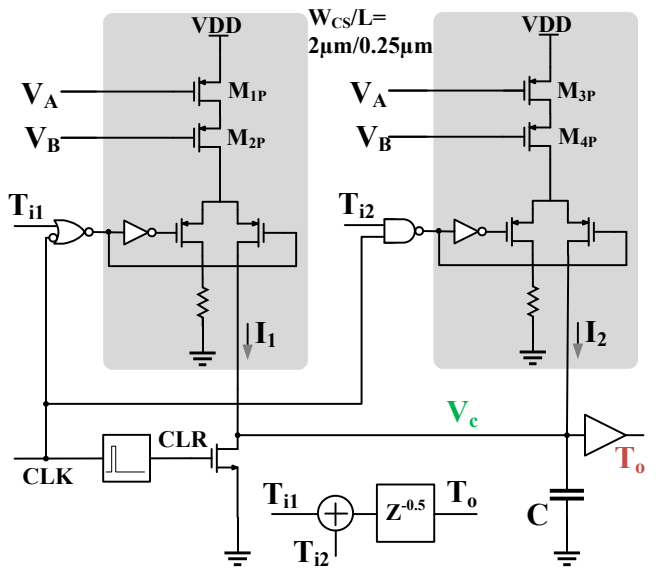

(b) Half-delay TA

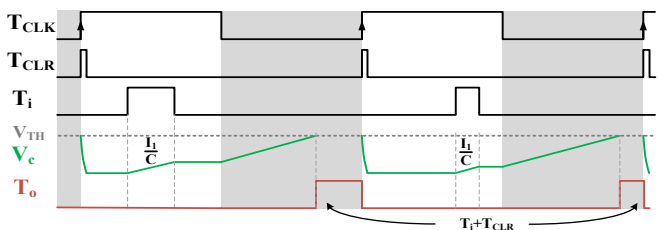

(c) Timing diagram of $\mathrm{T} R$

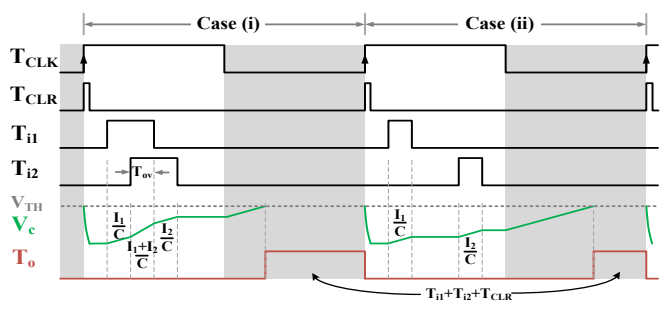

(d) Timing diagram of TA

Fig. 2: Circuit implementation and timing diagram of the half-delay time-domain register (TR) and time-domain adder (TA)

In case of a pseudo-differential architecture, $\Delta T_{o}=$ $\Delta T_{i} z^{-0.5}$. Although $\Delta T_{o}$ is not influenced by the offset $\left(T_{C L R}\right)$, it limits the maximum allowable input time difference $\left(T_{C L K} / 2-T_{C L R}\right)$. For the operation of the TR, $I_{1, \min }=$ $C V_{T H} /\left(\frac{T_{C L K}}{2}-T_{C L R}\right)$. With $\mathrm{C}=120 \mathrm{fF}, T_{C L R}=0.2 \mathrm{~ns}$ and $V_{T H}=V_{D D} / 2=0.6 \mathrm{~V}$, the current $I_{1, \min }$ equals to 15 $\mu A$ for a $T_{C L K}=10 \mathrm{~ns}$.

\section{B. Half-delay Time-domain Adder}

The half-delay time-domain adder (TA) (Fig. 2(b)) performs an addition of two pulse-time intervals $\left(T_{i 1}, T_{i 2}\right)$ at the input, and produces an $T_{o}$ with a half clock cycle delay. As shown in Fig. 2(d) when $C L K=1, C$ is charged using two matched cascoded current sources $\left(I_{1}, I_{2}\right)$, enabled by input time intervals $T_{i 1}$ and $T_{i 2}$ respectively.

For case (i), when $T_{i 1}$ overlaps with $T_{i 2}$, the total charge $\left(\Delta Q_{H}\right)$ stored in $C$ during sampling can be derived as, $\Delta Q_{H}=I_{1}\left(T_{i 1}-T_{o v}\right)+\left(I_{1}+I_{2}\right) T_{o v}+I_{2}\left(T_{i 2}-T_{o v}\right)$.

For case (ii), without any overlap between $T_{i 1}$ and $T_{i 2}$, the charge $\left(\Delta Q_{H}\right)$ stored in $C$ can be derived as, $\Delta Q_{H}=I_{1} T_{i 1}+$ $I_{2} T_{i 2}$. As $C L K$ becomes low, $I_{1}$ continues to charge $C$, and the charge stored equals to $\Delta Q_{L}=I_{1}\left(T_{C L K} / 2-T_{o}\right)$.

Similar to (1), it can be derived for both the above cases that, $T_{o}=\left(T_{i 1}+k T_{i 2}\right) z^{-0.5}+T_{C L R}$, where $k=I_{2} / I_{1}$. For a pseudo-differential architecture with matched current sources $\left(I_{1}=I_{2}\right)$ the output, $\Delta T_{o}=\left(\Delta T_{i 1}+\Delta T_{i 2}\right) z^{-0.5}$.

\section{Time-difference Detector}

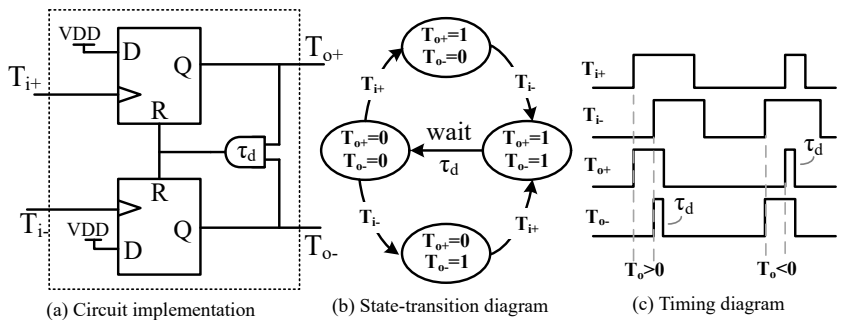

Fig. 3: Overview of the Time-difference detector (TD)
The time-difference detector (TD) (Fig. 3(a)) comprises 2 D-flipflops with an AND gate driving the RESET in feedback. It produces pulse-time intervals proportional to the time differences between the rising edges of the input signals. The AND-gate delay $\left(\tau_{d}\right)$ in feedback reduces the dead-zone and ensures a minimum width $\left(\tau_{d}\right)$ pulse at the outputs and thus improves the sensitivity of the circuit towards small $(<50 \mathrm{ps})$ input time differences.

Time-domain Differential Integrator: For matched current sources $I_{1}=I_{2}$, the overall system transfer function $\mathrm{H}(\mathrm{z})$ can be derived as, $H(z)=z^{-1} /\left(1-z^{-1}\right)$ and the gain and the phase of the integrator at the input frequency $\Omega_{i n}$ equals to $1 /\left(\Omega_{i n} T_{s}\right)$ and $-\pi / 2$ respectively.

\section{CAlibration Circuit}

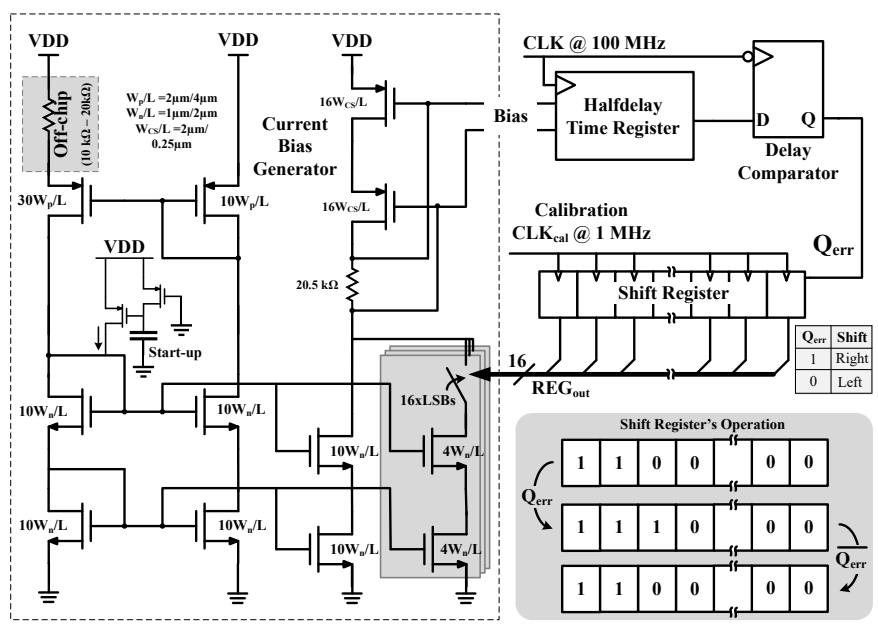

Fig. 4: Details of the calibration circuit for bias current

As discussed in section III, the charging current $\left(I_{1}\right)$ for the TRs and TAs need to be adjusted such that $\left(I_{1} / C\right)\left(T_{C L K} / 2-\right.$ $\left.T_{C L R}\right)=V_{T H}$ and it varies with changing temperature and different process conditions. In this case, the allowable 
range of the input time difference equals to $\pm\left(T_{C L K} / 2\right.$ $\left.T_{C L R}\right)$, which is limited by the variation of $V_{T H}$ and $I_{1}$. The calibration circuit operates similar to a DLL and it helps to adjust $I_{1}$ to the desired value. As shown in Fig. 4, the circuit comprises a half-delay TR, 16-cell shift register, and a bias circuit with a switchable 4-bit thermometer coded current reference. The off-chip resistor in the current bias generator is used for course tuning to accommodate larger variations across different process conditions.

The TR produces a pulse-time interval $\left(T_{p}=C \cdot V_{T H} / I_{1}\right)$ which is compared with respect to $T_{C L K} / 2$ using a D-flipflop. The D-flipflop produces an error output 1 , when $T_{p}+T_{C L R}>$ $T_{C L K} / 2$ and vice versa. For a positive error, the shift register shifts right and enables LSBs of the current reference to increase $I_{1}$ and reduce $T_{p}$ to adjust $T_{p} \rightarrow\left[T_{C L K} / 2-T_{C L R}\right]$. It starts with a RST pulse setting the output of the shift register to 8 and thereafter the calibration phase continues for a maximum of 8 clock cycles to adjust $I_{1}$ to the desired value. It ends when the output continues to toggle for 1 LSB-bit and hence a maximum $\pm 1 / 2$ LSB error is expected after calibration.

\section{Circuit Non-IDEAlities}

A brief investigation of the impact of the non-idealities is discussed as follows:

1) Current Mismatch: For a relative mismatch of $\epsilon_{I}$ between the current sources $\left(I_{1}, I_{2}\right)$, the feedback-path gain $a=\left(1 \pm \epsilon_{I}\right)$, and then $H(z)$ can be rewritten as, $H(z)=$ $\frac{z^{-1}}{1-\left(1 \pm \epsilon_{I}\right) z^{-1}}$. Furthermore, the gain and the phase can be estimated as $\frac{1}{\sqrt{\epsilon_{I}^{2}+\left(\Omega_{i n} T_{s}\right)}}$ and $\pm \tanh \frac{\Omega_{i n} T_{s}}{\epsilon_{I}}$ respectively. These distortions in the gain and the phase worsen in case of very small input frequencies (i.e, $\Omega_{i n} T_{s} \approx \epsilon_{I}$ ).

2) Non-linear Distortion: Assuming the PMOSs in the current sources operate in saturation during the charging phase, the cascoded current source can be modeled as a current of $I_{1, s a t}$ in parallel with $r_{o}$ [9]. Then the charging current $I_{c}(t)$ can be derived as, $I_{c}(t)=I_{c, s a t} \exp \left(-t / \tau_{o}\right)$, where $I_{c, \text { sat }}=\left(V D D / r_{o}+I_{1, s a t}\right)$ and $\tau_{o}=r_{o} C$. Now using Taylor series expansion for small inputs $\left(\left|T_{i}[n] / \tau_{o}\right|<1\right)$, the output of the single-ended TR can be approximated from (1) as,

$$
T_{o}[n+0.5] \approx\left(T_{i}[n]-\frac{T_{i}^{2}[n]}{\tau_{o}}+\ldots\right) \frac{I_{c, s a t}}{I_{1, s a t}}+T_{C L R}
$$

Although, $\tau_{o}$ is large in cascoded configuration, it reduces effectively due to the parasitics in high frequency $(100 \mathrm{MHz})$ applications, resulting in a non-uniform time-domain gain.

3) Charge Leakage: The charge injection from the gated PMOS switches, clock feed-through from activating input pulse-time signals generate voltage fluctuations across $\mathrm{C}$. In hold-state, finite impedance $\left(\mathrm{R}_{\text {off }}\right)$ of the discharging NMOS lead to leakage of charge stored in $C$. This introduces a voltage error $\left(\Delta v_{e}\right)$ causing a change in the threshold crossing time [9]. The resulting timing error $\left(\Delta t_{e}\right)$ can be estimated as, $\Delta t_{e}=\Delta v_{e} /$ slewrate $=C \Delta v_{e} / I_{1, \text { sat }}$.
4) Circuit Noise: The noise contributions of the cascoded PMOSs $\left(\mathrm{M}_{2 \mathrm{P}}, \mathrm{M}_{4 \mathrm{P}}\right)$ are negligible. The thermal noise from the PMOSs $\left(\mathrm{M}_{1 \mathrm{P}}, \mathrm{M}_{3 \mathrm{P}}\right)$ and the charging capacitors integrates over $\mathrm{C}$ as a wiener process and appear as brown voltage noise at the input of the comparator. As described in [9], [10] the resulting timing error due to thermal noise deposition can be estimated as,

$$
\overline{\Delta t_{t h}^{2}}=\frac{k T C}{I_{1, s a t}^{2}}+\frac{2 k T \gamma g_{m} t_{c h}}{I_{1, s a t}^{2}}
$$

where $t_{c h}=T_{C L K} / 2-T_{C L R}$ is the total charging time window. Similarly, the timing jitter due to flicker noise can be determined by considering the measurement time $\left[0, t_{m}\right]$ which limits the noise frequencies by the bandwidth $\left[f_{m}=\right.$ $\left.1 / t_{m}, \infty\right]$. The resulting timing error [10] can be derived as,

$$
\overline{\Delta t_{f l}^{2}}=\frac{K_{f} g_{m}^{2} t_{c h}^{2}}{I_{1, s a t}^{2} C_{o x} W L}\left(\frac{3}{2}-C i\left(2 \pi f_{m} t_{c h}\right)\right)
$$

where $C i(x)=-\int_{x}^{\infty} \frac{\cos x}{x} d x$ is cosine integral function.

Total Timing Error: The total timing error due to charge leakage and circuit noise can be estimated as, $\overline{\sigma_{t}^{2}}=\overline{\Delta t_{e}^{2}}+$ $\overline{\Delta t_{t h}^{2}}+\overline{\Delta t_{f l}^{2}}$, where $\overline{\Delta t_{e}}$ due to charge-leakage is dominant. Consequently the SNR in $\mathrm{dB}$ can be expressed as, $S N R=$ $10 \log _{10}\left(T_{i n, p p}^{2} / 8 \overline{\sigma_{t}^{2}}\right)$.

\section{Simulation Results}

The following results are obtained by analysing post-layout simulations with a supply voltage of $1.2 \mathrm{~V}$ and using a verilogA testbench for generating the input time differences. Fig. 5 shows the layouts of the proposed time-domain integrator and the calibration circuit. A symmetric layout of the integrator has been implemented to minimize potential differential offset arising due to mismatch conditions in the differential path.

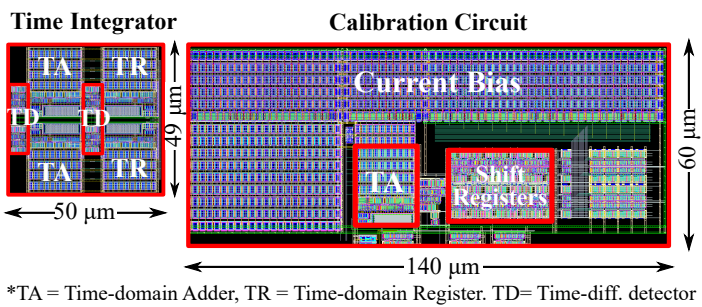

Fig. 5: The layouts of the proposed integrator and the calibration circuit.

The TD is simulated with respect to different input combinations $\left(T_{i 1}, T_{i 2}\right)$ within the range of $\pm 4 \mathrm{~ns}$ (at $\mathrm{TT}, 27^{\circ} \mathrm{C}$ ), and as shown in Fig. 6(a), linear transfer characteristics can be observed for each cases. Fig. 6(b) shows the values of the calibrated current $\left(I_{1}\right)$ with estimated error-bars. The calibration circuit adjusts $I_{1}$ around $15 \mu \mathrm{A}$ with an error of $\pm 0.7 \mu \mathrm{A}$, which has been verified at 3 different corners (FF, TT, SS) across temperature range of $-40^{\circ} \mathrm{C}$ to $80^{\circ} \mathrm{C}$.

The transient behavior of the proposed integrator is studied in Fig. 7(a). It can be observed that the integrator produces triangular output time differences when a square wave time differences $\left(T_{i n, p p}= \pm 1 \mathrm{~ns}, f_{i n}=7.69 \mathrm{MHz}\right)$ is applied 

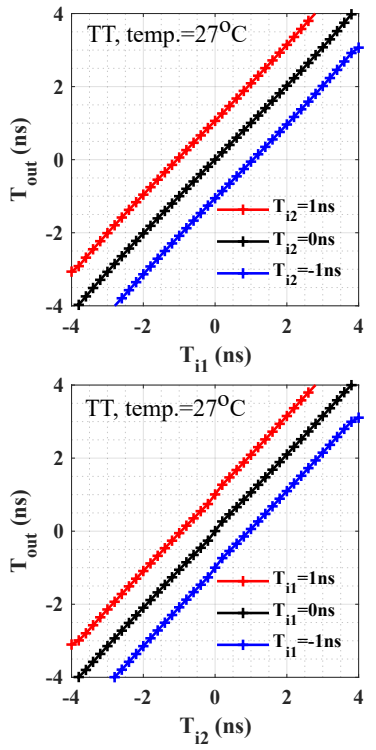

(a) $T_{o}$ vs $T_{i 1}$ and $T_{i 2}$
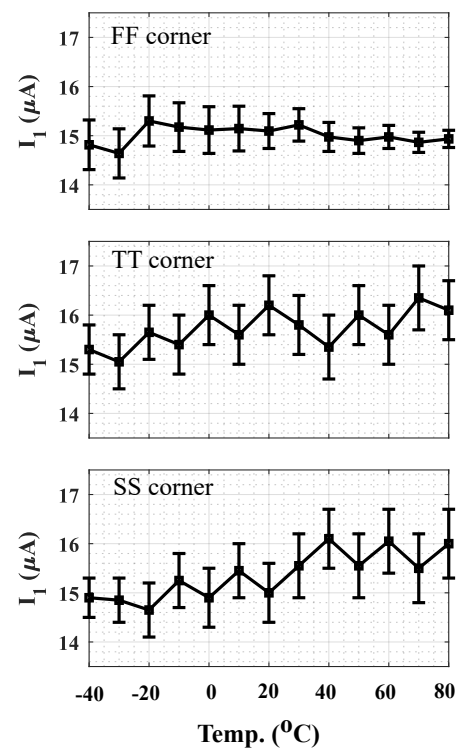

(b) Calibrated $I_{1}$ with estimated error bars.

Fig. 6: (a) Transfer characteristics of the TA and (b) response of the calibration circuit at different corners (FF,TT,SS).

at the input. In case of a sinusoidally varying input time difference $\left(T_{i n, p p}= \pm 1.5 \mathrm{~ns}, f_{i n}=6.2 \mathrm{MHz}\right)$, we can also observe a phase shift of $90^{\circ}$ between $T_{\text {in }}$ and $T_{\text {out }}$. As indicated in the frequency spectrums (Fig. 7(b)), the integrator produces a gain of $6.14 \mathrm{~dB}$ at the input signal frequency $\left(f_{i n}=6.2 \mathrm{MHz}\right)$. As evident from the Fig. 7(b), the nonidealities present in the circuit create harmonics (HD2 $=-42$ $\mathrm{dB}, \mathrm{HD} 3=-30 \mathrm{~dB}$ ) at the output. As compared to [7], the degradation in harmonic distortions can be attributed to larger signal bandwidth. However, these can easily be reduced if the time-domain integrator is used as filter in a $\Delta \Sigma$ modulator [8].

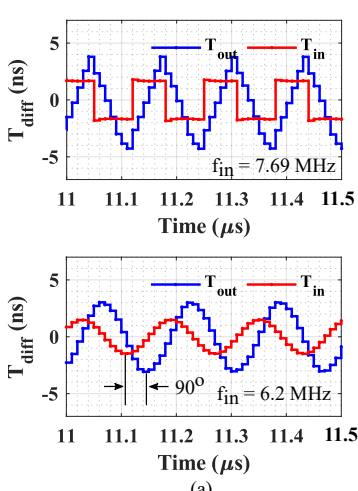

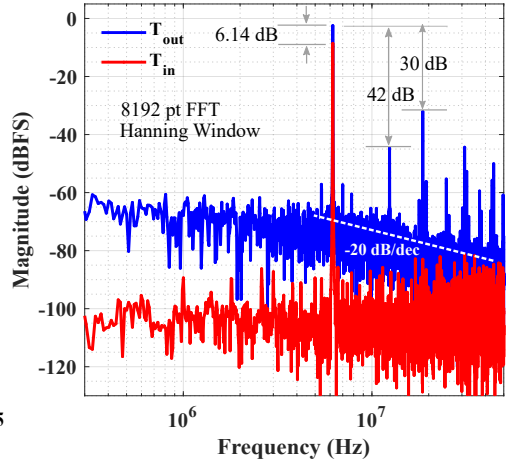

(b)

Fig. 7: (a) Output of the proposed integrator with a square wave input time differences (top), and a sinusoidal input time differences (bottom), (b) Input $\left(T_{i n}\right)$ and output $\left(T_{o u t}\right)$ spectrums of the time-domain integrator.

The Nyquist criteria requires a tradeoff between the timesignal amplitude and the system's bandwidth. Larger bandwidth implies smaller sampling window $\left(T_{C L K} / 2\right)$ resulting

in smaller achievable time-signals amplitudes. There, a larger power is spent to reduce the jitter induced time-domain noise and improve the SNR performance. A performance comparison of the proposed time-domain integrator with respect to others reported in literature is illustrated in Table. I.

TABLE I: Performance comparison with other time-domain integrators

\begin{tabular}{|c|c|c|c|c|}
\hline References & $\begin{array}{l}\text { Tech } \\
(\mathrm{nm})\end{array}$ & $\begin{array}{c}\mathrm{fs} \\
(\mathrm{MHz})\end{array}$ & $\begin{array}{l}\text { Power } \\
(\mu \mathrm{W})\end{array}$ & $\begin{array}{c}\left.\text { HD2[@ } f_{i n}\right] \\
(\mathrm{dB})\end{array}$ \\
\hline ISCAS'16 [6]* & 130 & 25 & 189.2 & $-42[@ 317 \mathrm{kHz}]$ \\
\hline Elec. Letters'18 [7]* & 65 & 50 & 152.7 & -50.9[@317 kHz] \\
\hline MWCAS'17 [11]* & 130 & 25 & 101.6 & -22[@415 kHz] \\
\hline This work ${ }^{\dagger}$ & 65 & 100 & 740 & -42[@6.2 MHz] \\
\hline
\end{tabular}

\section{CONCLUSION}

A pseudo-differential time-domain integrator is proposed which performs accumulation of time information using charge-based time-domain register and adders. The differential architecture helps to process bipolar time information and is particularly suited for applications as loop filters in noise shaping $\Delta \Sigma$ TDCs [3], [4] and $\Delta \Sigma$ modulators for sensing applications. The architecture can also easily be extended to realize higher order IIR and FIR filters for time-domain signal processing [1].

\section{REFERENCES}

[1] M. Abdelfattah and G. W. Roberts, "All-Digital Time-Mode Direct-Form All-Pole Biquadratic Filter Realization," IEEE Transactions on Circuits and Systems II: Express Briefs, vol. 64, no. 11, pp. 1262-1266, 2017.

[2] K. Kim, W. Yu and S. Cho, "A 9 bit, 1.12 ps Resolution 2.5 b/Stage Pipelined Time-to-Digital Converter in $65 \mathrm{~nm}$ CMOS Using TimeRegister," in IEEE Journal of Solid-State Circuits, vol. 49, no. 4, pp. 1007-1016, April 2014.

[3] S. Ziabakhsh, G. Gagnon, and G. W. Roberts, "A Second-Order Bandpass $\Delta \Sigma$ Time-to-Digital Converter With Negative Time-Mode Feedback," IEEE Transactions on Circuits and Systems I: Regular Papers, vol. 66, no. 4, pp. 1355-1368, 2019.

[4] Y. Wu, P. Lu, and R. B. Staszewski, "A Time-Domain 147fsrms 2.5-MHz Bandwidth Two-Step Flash-MASH 1-1-1 Time-to-Digital Converter with Third-Order Noise-Shaping and Mismatch Correction," IEEE Transactions on Circuits and Systems I: Regular Papers, pp. 1-14, 2020.

[5] Y. J. Park and F. Yuan, "All-Digital $\Delta \Sigma$ TDC With Differential BiDirectional Gated-Delay-Line Time Integrator," in 2017 IEEE 60th International Midwest Symposium on Circuits and Systems (MWSCAS), 2017, pp. 1513-1516.

[6] Y. J. Park, D. Jarrett-Amor, and F. Yuan, "Time Integrator for MixedMode Signal Processing," in 2016 IEEE International Symposium on Circuits and Systems (ISCAS), 2016, pp. 826-829.

[7] K. Zhu, J. Feng, Y. Lyu, and A. He, "High-precision differential time integrator based on time adder," Electronics Letters, vol. 54, no. 22, pp. 1268-1270, 2018.

[8] H. Kwon, J. Lee, J. Sim, and H. Park, "A high-gain wide-input-range time amplifier with an open-loop architecture and a gain equal to current bias ratio," in IEEE Asian Solid-State Circuits Conference 2011, 2011, pp. 325-328.

[9] F. Yuan and P. Parekh, "Analysis and Design of an All-Digital $\Delta \Sigma$ TDC via Time-Mode Signal Processing," IEEE Transactions on Circuits and Systems II: Express Briefs, vol. 67, no. 6, pp. 994-998, 2020.

[10] S. Ziabakhsh, G. Gagnon, and G. W. Roberts, "The Peak-SNR Performances of Voltage-Mode versus Time-Mode Circuits," IEEE Transactions on Circuits and Systems II: Express Briefs, vol. 65, no. 12, pp. 18691873, 2018.

[11] Y. J. Park and F. Yuan, "1-1 MASH $\Delta \Sigma$ Time-to-Digital Converter With Differential Cascode Time Integrator," in 2017 IEEE 60th International Midwest Symposium on Circuits and Systems (MWSCAS), 2017, pp. 1005-1008. 